\title{
Effect of mega voltage energy on dose enhancement in phantom study by using gold nanoparticle polymer gel dosimeter
}

\author{
Gholamreza Atae ${ }^{1}$, Seyed Rabee Mahdavi ${ }^{2}$, Akram Mohammadi Nokhandani ${ }^{1}$, \\ Syede Mahsa Taheri Otaghsara ${ }^{1}$, Mahboubeh Khadem Abolfazli ${ }^{1, *}$ \\ ${ }^{1}$ Babol University of Medical Science, paramedical school, Babol, Iran \\ ${ }^{2}$ Iran University of Medical Science, Tehran, Iran
}

\section{Email address:}

golamrezaatae@yahoo.com (G. Atae), srmahdavi@hotmail.com (S. R. Mahdavi), akram433mohammadi@yahoo.com (A. M. Nokhandani), mahboubeh.khadem@yahoo.com (M. K. Abolfazli)

\section{To cite this article:}

Gholamreza Atae, Seyed Rabee Mahdavi, Akram Mohammadi Nokhandani, Syede Mahsa Taheri Otaghsara, Mahboubeh Khadem Abolfazli. Effect of Mega Voltage Energy on Dose Enhancement in Phantom Study by Using Gold Nanoparticle Polymer Gel Dosimeter. International Journal of Biomedical Science and Engineering. Vol. 3, No. 1, 2015, pp. 1-4. doi: 10.11648/j.ijbse.20150301.11

\begin{abstract}
Background: Polymer gels beam sensitive are valuable and confident for measurement of 3D dose distributions. One of the special features of this type of dosimeters is their tissue equivalence, which makes them appropriate for the investigation of dose enhancement with contrast agents with high atomic number. Objective: The purpose of this study is an investigating of dose enhancement dependent in energy within the gel medium with used of conformal distribution gold nanoparticle as contrast agents by high atomic number material. Methods: In this work, gold nanoparticles (GNPs) of 50nm diameter with $0.1 \mathrm{~m}$ concentration embedded in gel and irradiated by different megavoltage energy of $18 \mathrm{MVand} 6 \mathrm{MV}$ photon beam. Then GN- MAGICA and MAGICA dose response curves compare and achieved dose enhancement. Results: Experimental results have shown dose enhancement factor of $6 \mathrm{MV}$ and $18 \mathrm{MV}$ in $0.1 \mathrm{mM}$, concentration is 1.74 and 1.04 respectively. Conclusion: The results showed that by adding of gold nanoparticles to the MAGICA polymer gel absorbed dose is increased. The levels of polymerization of irradiated gels with and without AuNPs in energy 6MV is more than energy 18MV. It seems that because of the dominance of photoelectric effect at low energies and pair production effect at high energies.
\end{abstract}

Keywords: Dose Enhancement, Polymer Gel Dosimeter, Gold Nanoparticles

\section{Introduction}

Primary goal of radiotherapy research is a optimizing the therapeutic ratio, the damage to tumor cells compared to normal tissue. In clinical practice, normal tissue tolerance is often the limiting factor in setting the treatment dose (1). An alternative method of enhancing the therapeutic ratio is through a binary treatment involving the preferential uptake of a dose enhancer or 'radiosensitizer' within the tumor. One of the famous radio sensitizer is a gold nanoparticle. Application of gold nanoparticle (AuNPs) in dose enhancement in radiation therapy has been studied in the last decade. Although several studies have shown the effect of high atomic number materials used in radiology such as iodinated contrast media on the dose enhancement, development of nano-scaled material with higher penetrability into cells as well as cell nucleus increased scientists' interests in applying these materials in radiation therapy $(2,3)$.

Furthermore, in vivo and in vitro studies have demonstrated that gold nanoparticles (AuNps) are ideal radiation-enhancing agents for cancer radiation therapy (4-6). However, the major limitation of these methods is the inability to perform in-depth investigations into the influence of these metal nanoparticles on the 3-dimensional (3D) spatial aspects of the radiation dose distribution, which includes doses to surrounding critical structures, and the effects of particle type, morphology, and surface chemistry on radiation therapy dose enhancement. Therefore, there is an urgent need to develop an independent experimental approach to answer these questions. One potential method is to use $3 \mathrm{D}$ dosimeters to better understand the radiation-metal nanoparticle interaction outcomes. 3D polymer gel dosimeters are fabricated from radiation-sensitive materials that change their properties when absorbing a 
radiation dose, and they have been developed to model experimentally and improve dose delivery (7). Each type of dosimeter interacts differently with radiation, and they have unique methods of recording the radiation dose distribution in $3 \mathrm{D}$ compared with conventional ion chambers and 2-dimensional dosimeters such as films, which are limited to point or planar measurements $(7,8)$. However, attempts to exploit current $3 \mathrm{D}$ radiation dosimeters in metal nanoparticle dosimetry are yet to be documented.

In this study, we have developed a technique of measuring depth dose enhancement by using MAGICA doped with AuNPs. Our measurement aims to determine the feasibility of using MAGICA-AuNPs as a dosimeter. We report the depth dose enhancement from clinical photon beams from a linear accelerator.

\section{Materials and Method}

\subsection{Choice Size and Preparation of AuNPS}

Gold nanoparticle were selected for this initial investigation because their enhancing properties have been demonstrated in several imaging and therapeutic studies (9-11). In addition, a recent study has shown that maximum cellular uptake and dose enhancement occurs with 50-nm diameter particles(10); therefore 50-nm diameter particles were selected for this study however, because 50-nm AuNPs are not readily available commercially it was necessary to fabricate such particle. Therefore, AuNPs have obtained from PNF Co, Iran, Tehran (Payam Avaran Nanofardanegar). In PNF Company, nanoparticles are produced by applying and extra high electric voltage and current, and the primary bulk wire with $0.1 \mathrm{~mm}$ diameter is then converted into nanoparticles via pulse explosive process.

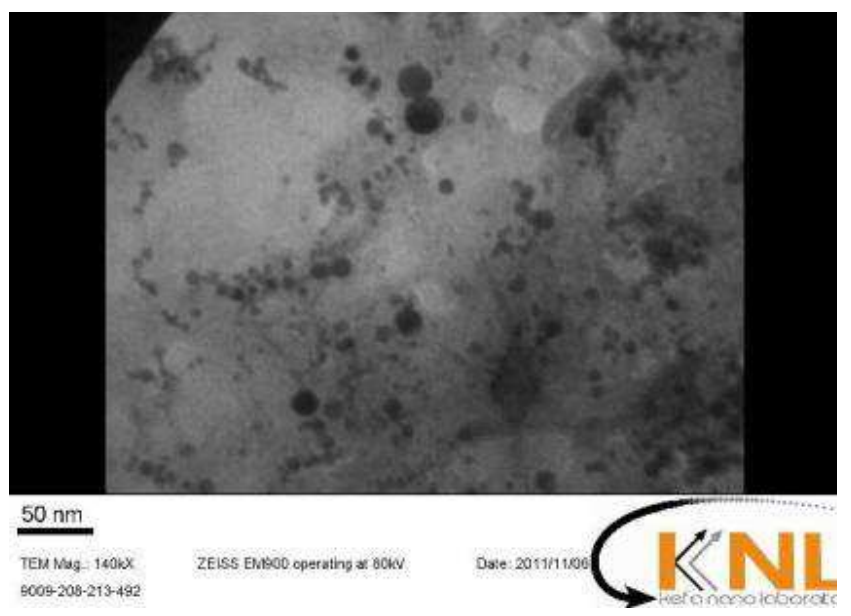

Figure 1. The gold nanoparticles were characterized using diameter of $50 \mathrm{~nm}$ with concentration $7 \mathrm{mg} / \mathrm{ml}$ (7000ppm)

\subsection{MAGICA Gel Dosimeter Fabrication}

The MAGICA polymer gels used in this study were prepared using methods documented previously $(12,13)$. The gel solution consists of water HPLC de-ionized, gelatin (typeA, bloom 250), Agarose, hydroquinone, methacrylic acid,
$\mathrm{CuSo}_{4}$ and Ascorbic acid.

For preparation of MAGICA, first water divided into 5 flasck of varying size, ready for dissolving each substance. Gelatin added into about $64 \%$ of total HPLC de-ionized water. Gelatin allowed swelling for about an hour. Then the solution stirred and heated to about $50^{\circ} \mathrm{C}$ until a clear solution obtained. When the temperature of gelatin solution reach near $40^{\circ} \mathrm{C}$, Agarose added to about $25 \%$ of warm water up to $500^{\circ} \mathrm{C}$. Agarose solution stirred and heated to about $90^{\circ} \mathrm{C}$ at which Agarose thoroughly dissolved. Both solutions were allowed to cool. When both solution cooled to an equal temperature about $47^{\circ} \mathrm{C}$, agarose solution was added to the gelatin solution and stirring continued. Stirring never stopped before the end of fabrication. At $37^{\circ} \mathrm{C}$, Hydroquinone, this solved in about $5 \%$ water, mass added to the mixture.

The remaining of water were divided into two portions and in each portion Ascorbic acid and capper(II) sulphate were dissolved after being weighted these two chemicals, which together play the role of oxygen scavenger, were added to the mixture when temperature declined to about $35^{\circ} \mathrm{C}$. Methacrylic acid was added at same temperature. The gel solution was two separated into two batches. The AuNPs concentration of $0.1 \mathrm{mM}$ added to the batch. The AuNPs were observed to mix homogeneously in the gel. One batch of whit out AuNPs served as a control. The gel was the quickly poured into separates vials. The vials wrapped with Para-film to reduce the effects of oxygen. All the samples were placed in the refrigerator in $4^{`} \mathrm{C}$ temperature overnight for the gel to set.

\subsection{Irradiation}

Samples from batches were irradiated with Mega voltage $\mathrm{X}$-rays. Mega voltage irradiation was carried out using a $6 \mathrm{MV}$ and $18 \mathrm{MV}$ linear accelerator (Varian) various radiation doses were delivered $(0,2,4$ and $6 \mathrm{~Gy})$. A single-fraction irradiation with a dose rate of $1.13 \mathrm{~Gy} / \mathrm{min}$, a $25 \times 25 \mathrm{~cm}$ field size was set.

\subsection{MRI Scanning}

Irradiated and non-Irradiated gel samples were scanned using a $0.5 \mathrm{~T}$ MRI scanner (Philips, Intera), to measure spin-spin relaxation time of the free protons using a head coil. A fast- spin echo sequence was used with following parameters: field of view $256 \times 256$ matrix, slice thickness $=3 \mathrm{~mm}$ (calibration vials) and $5 \mathrm{~mm}$ (gold nano MAGICA vials), effective echo time TE $=20-160 \mathrm{~ms}$, repetition time $(\mathrm{TR})=1500 \mathrm{~ms}$, number of slice $=5$, at least 24 hour elapsed after irradiation prior to imaging to allow for polymerization. All the samples scanned of $21^{\circ} \mathrm{C}$ temperature.

\subsection{Date Analysis}

Analysis of the image was performed using MATLAB soft ware (version 7.3.0.26) (The math works Inc, Natick, Massachusetts, USA). The program examined the data before analyzing it to determine the region of interest $T_{2}$ values were calculated and formed $\mathrm{T}_{2}$ maps on a pixel-by-pixel basis. The levels of polymerization of irradiated gels with and without AuNPs compared by calculating the $R_{2}=\left(1 / T_{2}\right)$. 


\subsection{Statistical Analysis}

This study presents result (mean values \pm standard deviation) from 3 independent experiments. One-way analysis of variance (ANOVA) used to determine the significant of difference between the control and experimental group. A difference was considered to be statistically significant when $\mathrm{P}<0.05$ statistical analysis was performed with the SPSS19 soft ware.

\section{Results and Discussion}

MAGICA response to the $6 \mathrm{MV}$ and $18 \mathrm{MV} \mathrm{x}$-ray beam was characterized by $\mathrm{R}_{2}$ signal relation to Dose.

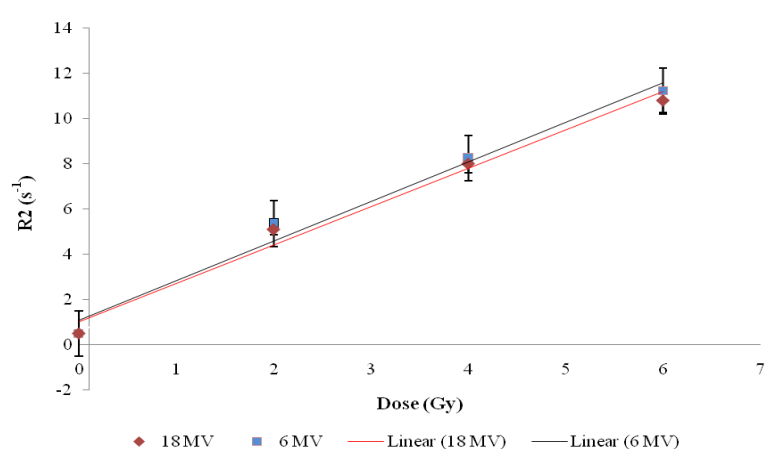

Figure 2. MAGICA polymer gel dosimeter $R_{2}$ response on absorb dose in range of $0-6 G y$.

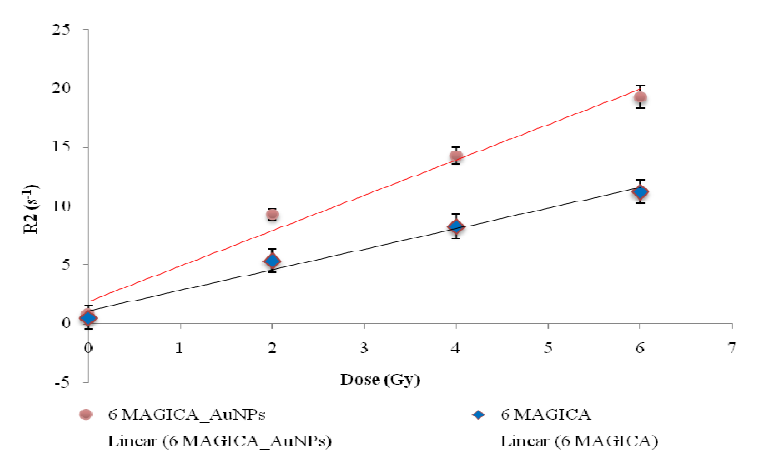

A

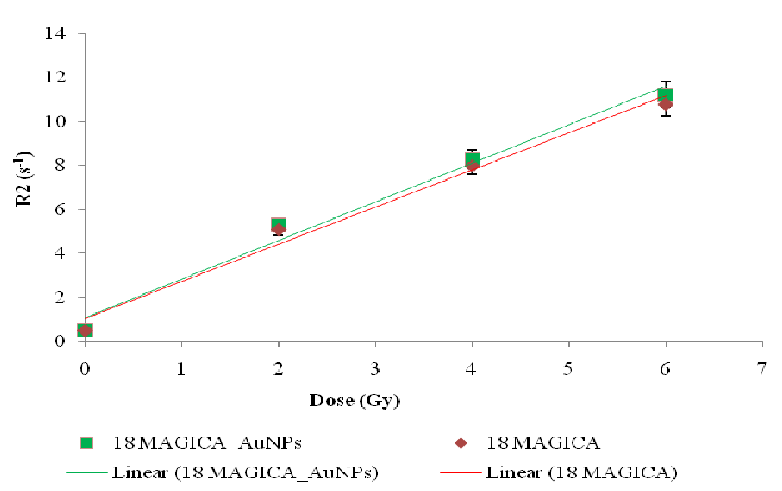

B

Figure 3. $A, B$ shows the dose-response relationship for two different experiments using gels irradiated by a $6 \mathrm{MV}$ and $18 \mathrm{MV}$ photon beam.
The dose response slopes for $\mathrm{R}_{2}$ versus delivered X-ray dose for MAGICA-AuNPs and pure MAGICA were calculated. The ratio of these slopes was taken as the dose enhancement factor (DEF). Figures $3 \mathrm{a}, \mathrm{b}$ shows the dose-response relationship for two different experiments using gels irradiated by an $18 \mathrm{MV}$ and $6 \mathrm{MV}$ photon beam. The average DEF obtained for 6MV was 1.74 with inter batch standard deviation of 0.028 and much lower DEF of 1.04 obtained for $18 \mathrm{MV}$ photon beams. The outcome of this work demonstrates the feasibility of introducing AuNPs into polymer gel dosimeters and the ability to scan them with the MRI scanner in the same way as standard polymer gel samples that do not contain metallic particles. The doping of polymer gels with metallic atoms such as iodine, gadolinium and recently AuNPs has been documented demonstrating that the dose enhancement caused by the presence such metallic atoms can be separated from the dose deposited in the gels.

The calibration curve shows dose response MAGICA gel independent from energy and almost linear within the range of $0-6 \mathrm{~Gy}$.

\section{Conclusion}

Generally, dose enhancement extracted from the comparison of the dose measured by gels with and without inclusion of the metallic atoms. The measured dose is related to the dose indicator factor " $\mathrm{T}_{2}$ " of the MRI parameter for the two gels. However, inclusion of contrast agent or nano particles into polymer gel must take into account its chemical properties that will eventually affect the dose measured. Iodinated compounds have observed to interfere with the interaction processes between the ions and monomers (7).

Two major advantage of polymer gel dosimeter were their ability to determine integrated $3 \mathrm{D}$ dose distribution as well as their ability to form in different shapes (4). In fact, polymer gel dosimeters were monomers, which distributed in a gelling matrix. Ionizing irradiation coverts these monomers to polymers via distinguished mechanism (7). The polymerization degree depends on absorbed dose in gel dosimeter. After polymerization, magnetic properties of polymer surrounding protons are changed (4).These changes could be exhibited by magnetic resonance imaging. The spin-spin relaxation rate $R_{2}=1 / T_{2}$ is related to absorbed dose, which was delivered to a gel phantom (6-8). One of the polymer gels advantages is able directly to measure the effects of contrasts agents or metallic radiation dose enhancers such as iodine and gold nanoparticles (GNPs) inside the dosimeter. In gel dosimeters, contrasts agents may have uniform dispersion within the dosimeter and therefore the effects of this material can directly quantified. Physical measurement of the dose enhancement produced by high $\mathrm{Z}$ materials with other types of radiation dosimeters, such as a film and ionization chambers are quite complicated. According to experimental and simulation results, by adding of gold nanoparticles to the MAGICA polymer gel absorbed dose is increased. In this study, a comparison of the MAGICA polymer gel absorbed dose with and without the presence of 
$0.1 \mathrm{mM}$ gold nanoparticles in different energy (6 and $18 \mathrm{MV}$ ) made. The radiation dose rate in linear accelerator was within the $0-6 \mathrm{~Gy}$. The $\mathrm{R}_{2}$ Dose graphs plotted for that energy. In the graphs to the conclusion that the parameter R2 (The levels of polymerization of irradiated gels with and without AuNPs) in energy $6 \mathrm{MV}$ is more than energy $18 \mathrm{MV}$. It seems that because of the dominance of photoelectric effect at low energies and pair production effect at high energies.

\section{References}

[1] Burnet NG, Wurm R, Nyman J, Peacock JH (1996) Normal tissue radiosensitivity - How important is it? Clinc Oncol 8:25-34.

[2] Raetorius NP, Mandal TK. Engineered nanoparticles in cancer therapy.Recent Pat Drug Deliv Formul2007;1:37-51

[3] Mukherjee P, Bhattacharya R, Bone N, Lee YK, Patra CR, Wang S, et al. Potential therapeutic application of gold nanoparticles in B-chronic lymphocytic leukemia (BCLL): enhancing apoptosis.J Nanobiotechnol2007;5:4.

[4] Rahman WN, Bishara N, Ackerly T, et al. Enhancement of radiation effects by gold nanoparticles for superficial radiation therapy.Nano-medicine2009;5:136-142.

[5] Hainfeld JF, Slatkin DN, Smilowitz HM. The use of gold nanoparticles to enhance radiotherapy in mice.Phys Med Biol2004;49:N309-N315
[6] Jain S, Coulter JA, Hounsell AR, et al. Cell-specific radiosensitization by gold nanoparticles at megavoltage radiation energies. Int $\mathrm{J}$ Radiat Oncol Biol Phys2011;79:531-539.

[7] Baldock C, De Deene Y, Doran S, et al. Polymer gel dosimetry. Phys Med Biol2010;55:R1-R63.

[8] Doran SJ. The history and principles of chemical dosimetry for 3-D radiation fields: gels, polymers and plastics. Appl Radiat Isotopes 2009; 67:393-398.

[9] Butterworth KT, Coulter JA, Jain S, et al. Evaluation of cytotoxicityand radiation enhancement using $1.9 \mathrm{~nm}$ gold particles: potentialapplication for cancer thpy. Nanotechnology 2010; 21: 295101.

[10] Chithrani DB, Jelveh S, Jalali F, et al. Gold nanoparticles as radiation sensitizers in cancer therapy. Radiat Res2010; 173:719-728.

[11] Rahman WN, Bishara N, Ackerly T, et al. Enhancement of radiationeffects by gold nanoparticles for superficial radiation therapy.Nano-medicine2009;5:136-142.

[12] Zahmatkesh MH, Kousari R, Akhlaghpour S, Bagheri SA (2004) MRI gel dosimetry with methacrylic acid, ascor-bic acid, hydroquinon and copper in agarose (MAGICA) gel. In: Preliminary Proceedings of DOSGEL 2004; Ghent, Belgium.

[13] Gustavsson H (2004) Radiotherapy gel dosimetry, development and application of normoxic polymer gels, [Ph.D Thesis], Malmo University Hospital, Lund University, Lund, Sweden 